\title{
A Note on the Paper "Optimality Conditions for Optimistic Bilevel Programming Problem Using Convexifactors"
}

\section{Bhawna Kohli ${ }^{1}$}

Received: 20 August 2018 / Accepted: 9 December 2018 / Published online: 18 December 2018

c) Springer Science+Business Media, LLC, part of Springer Nature 2018

\section{Abstract}

This note concerns about the conclusion of a lemma of a published paper of this journal.

Keywords Bilevel programming problem · Convexifactors · Value function . Constraint qualifications · Optimality conditions

\section{Introduction}

Bilevel programming problems are hierarchical problems of two decision makers, where the first decision maker, or the leader, gives his choice and the second decision maker, or the follower, reacts optimally on it and the optimal solution is then used to calculate the objective function value of the leader's problem. Kohli [1] introduced two versions of nonsmooth Abadie constraint qualification and employed the weaker version to develop Karush-Kuhn-Tucker type necessary optimality conditions for optimistic bilevel programming problem with convex and nonconvex lower-level problems, using an upper estimate of Clarke subdifferential of value function and the concept of convexifactor. The conclusion of [1, Lemma 5.2] is not correct. Here, we correct its conclusion and also the conclusion of the necessary optimality conditions, which makes use of it.

\section{Optimality Conditions}

We begin this section by providing a change in the conclusion of the lemma.

Remark 2.1 In the conclusion of [1, Lemma 5.2], $0 \in B-A$ should be replaced by $0 \in B+A$ [2, Lemma 2.1].

Bhawna Kohli

bhawna_kohli@rediffmail.com

1 Department of Mathematics, P.G.D.A.V. College, University of Delhi, New Delhi 110065, India 
Remark 2.2 [1, Theorem 5.1] makes use of [1, Lemma 5.2]. There is a change from '-'sign to ' + ' sign after conv $\partial^{*} F(\bar{x}, \bar{y})$ in the necessary optimality condition (i) ([1, Theorem 5.1]) and necessary optimality condition (11) (Page 646) after applying [1, Lemma 5.2] with the above mentioned change to the sets coneconv $A$ and $\operatorname{conv} \partial^{*} F(\bar{x}, \bar{y})$.

Remark 2.3 In [1, Example 5.4] and [1, Example 5.5], we can take $G(x, y)$ from [1, Example 5.6] to achieve our target.

Remark 2.4 In [1, Example 5.6], we can take $G(x, y)$ from [1, Example 5.2] to achieve our target.

\section{Conclusions}

Kohli [1] investigated bilevel programming problem. Here, we correct the conclusion of [1, Lemma 5.2] as well as the conclusion of optimality conditions, which makes use of it.

Acknowledgements The author is grateful to the Editor-in-Chief for his constant guidance.

\section{References}

1. Kohli, B.: Optimality conditions for optimistic bilevel programming problem using convexifactors. J. Optim. Theory Appl. 152(3), 632-651 (2012)

2. Kohli, B.: Necessary and sufficient optimality conditions using convexifactors for mathematical programs with equilibrium constraints. Rairo Oper. Res. (2018). https://doi.org/10.1051/ro/2018084

Publisher's Note Springer Nature remains neutral with regard to jurisdictional claims in published maps and institutional affiliations. 